\title{
Antioxidant activity relationship of phenolic compounds in Hypericum perforatum L.
}

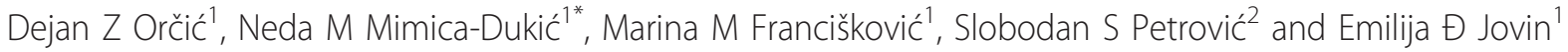

\begin{abstract}
Background: The St John's Wort (Hypericum perforatum; Clusiaceae) has been used in traditional and modern medicine for a long time due to its high content of biologically active phenolics. The purpose of this work was to develop a method for their fractionation and identification, and to determine the most active antioxidant compounds in plant extract.

Results: An LC-MS method which enables fast qualitative and semiquantitative analysis was developed. The composition determined is in agreement with the previous results, where 6 flavonoids, 4 naphthodianthrones and 4 phloroglucinols have been identified. Significant antioxidant activity was determined for most of the fractions by DPPH assay (the lowest $I C_{50}$ of $\left.0.52 \mu \mathrm{g} / \mathrm{ml}\right)$, NO scavenging $(6.11 \mu \mathrm{g} / \mathrm{ml})$, superoxide scavenging $(1.86 \mu \mathrm{g} / \mathrm{ml})$, lipid peroxidation $(0.0079 \mu \mathrm{g} / \mathrm{ml})$ and FRAP (the highest reduction capacity of $104 \mathrm{mg}$ Fe equivalents $/ \mathrm{g}$ ) assays.

Conclusion: LC-MS technique has been successfully applied for a quick separation and identification of the major components of $\mathrm{H}$. perforatum fractions. Majority of the fractions analyzed have expressed a very high antioxidative activity when compared to synthetic antioxidants. The antioxidant activity could be attributed to flavonoids and phenolic acids, while phloroglucinols and naphthodianthrones showed no significant activity. It is demonstrated that it is possible to obtain, by fractionation, $H$. perforatum preparations with significantly increased phloroglucinolsto-naphthodianthrones ratio (up to 95:5).
\end{abstract}

\section{Background}

Hypericum perforatum L. (St. John's wort) is a representative of the Clusiaceae family with confirmed therapeutic effects on burns, bruises, swelling, anxiety, mild to moderate depression [1], antidepressant, antiviral, wound healing, analgesic, hepatoprotective, antioxidant and antimicrobial activity [2-5]. Hyperforin is believed to be the major constituent responsible for the antidepressant activity, although discrepancies in the doseresponse relationship imply that other constituents are also involved [6]. Naphthodianthrones, especially hypericin-like compounds, are shown to posses a strong antiviral effect [7]. Flavonoids are of a special interest due to their antioxidative properties i.e. excellent radical scavenging ability [6].

\footnotetext{
* Correspondence: neda.mimica-dukic@dh.uns.ac.rs

${ }^{1}$ Department of Chemistry, Biochemistry and Environmental Protection, University of Novi Sad Faculty of Sciences, Trg Dositeja Obradovića 3, Novi Sad, Serbia

Full list of author information is available at the end of the article
}

Reactive oxygen species (ROS) have been implicated in pathogenesis of many diseases, including cancer, mutagenesis, Alzheimer's, AIDS, etc. [8]. Many synthetic antioxidants are currently in use; nevertheless, there is a growing evidence of consumer preference for natural antioxidants because of their potentially lower toxicity. $H$. perforatum extracts are already proven to inhibit lipid peroxidation in vivo [5].

Previous results [9] demonstrated that $H$. perforatum fractions, obtained by successive liquid-liquid extractions with solvents of different polarities, have different epileptic activities (ranging from inhibitory to stimulatory), and that this approach can point out to compounds responsible for pharmacological activity. In this work eight fractions of $H$. perforatum, obtained by an externally developed method of fractionation devised to separate main constituents of the plant, were examined. The chemical composition of all extracts has been analyzed by rapid-resolution liquid chromatography, as opposed to the previously used classical high-performance liquid chromatography [10-18]. Antioxidant activity has been 
assessed by scavenging DPPH radical, NO radical, superoxide anion radical, FRAP test, and lipid peroxidation using a linseed oil as a substrate. Where possible, synthetic antioxidants BHT (3,5-di-tert-butyl-4-hydroxytoluene) and BHA (2-tert-butyl-4-hydroxyanisole) were used as standards. An attempt was made to correlate chemical composition of the extracts with its antioxidant activity and determine which group of biomolecules is the most potent.

\section{Results}

\section{LC-MS analysis}

LC-MS technique has been successfully applied for a quick separation and identification of the major components of $H$. perforatum fractions. Detected compounds are representatives of three groups: flavonoids, naphthodianthrones and phloroglucinols.

Peaks identification was done by comparing the mass spectra and retention times with already published data [17]. Four naphthodianthrones (protopseudohypericin, pseudohypericin, protohypericin and hypericin) and four phloroglucinols (hyperfirin, adhyperfirin, hyperforin, adhyperforin) were identified together with several flavonoids (hyperoside, rutin, quercitrin and quercetin) and a small amount of caffeoylquinic acid (Table 1). The grouping of compounds by classes is observed, with flavonoids and phenolic acids eluting between 0 and 2.5 min, naphthodianthrones in 2.5-4.7 min, and phloroglucinols in 4.7-6.5 min interval (Figure 1). The relative abundance of each group is determined by the normalization method, as an area percent of all peaks with similar spectra eluting within a given retention time range

Table 1 Retention times and $[\mathrm{M}-\mathrm{H}]^{-}$ions of identified peaks

\begin{tabular}{|c|c|c|c|}
\hline Peak & $\begin{array}{c}t_{R} \\
{[\mathrm{~min}]}\end{array}$ & $\begin{array}{c}{[\mathrm{M}-\mathrm{H}]^{-},} \\
\mathrm{m} / \mathrm{z}\end{array}$ & Compound \\
\hline \multirow[t]{3}{*}{1} & 0.13 & 353 & caffeoylquinic acid \\
\hline & & 463 & $\begin{array}{l}\text { quercetin-3-O- } \beta \text {-D-galactopyranoside } \\
\text { (hyperoside) }\end{array}$ \\
\hline & & 609 & quercetin-3-O-rutinoside (rutin) \\
\hline 2 & 0.16 & 447 & $\begin{array}{l}\text { quercetin-3-O- } \alpha \text {-L-rhamnopyranoside } \\
\text { (quercitrin) }\end{array}$ \\
\hline 3 & 0.34 & 301 & quercetin \\
\hline 4 & 0.96 & 537 & 13, ||8-biapigenin \\
\hline 5 & 1.47 & 537 & amentoflavone (I3', II8-biapigenin) \\
\hline 6 & 2.75 & 521 & protopseudohypericin \\
\hline 7 & 2.87 & 519 & pseudohypericin \\
\hline 8 & 3.69 & 505 & protohypericin \\
\hline 9 & 3.83 & 503 & hypericin \\
\hline 10 & 4.80 & 467 & hyperfirin \\
\hline 11 & 4.95 & 481 & adhyperfirin \\
\hline 12 & 5.53 & 535 & hyperforin \\
\hline 13 & 5.68 & 549 & adhyperforin \\
\hline
\end{tabular}

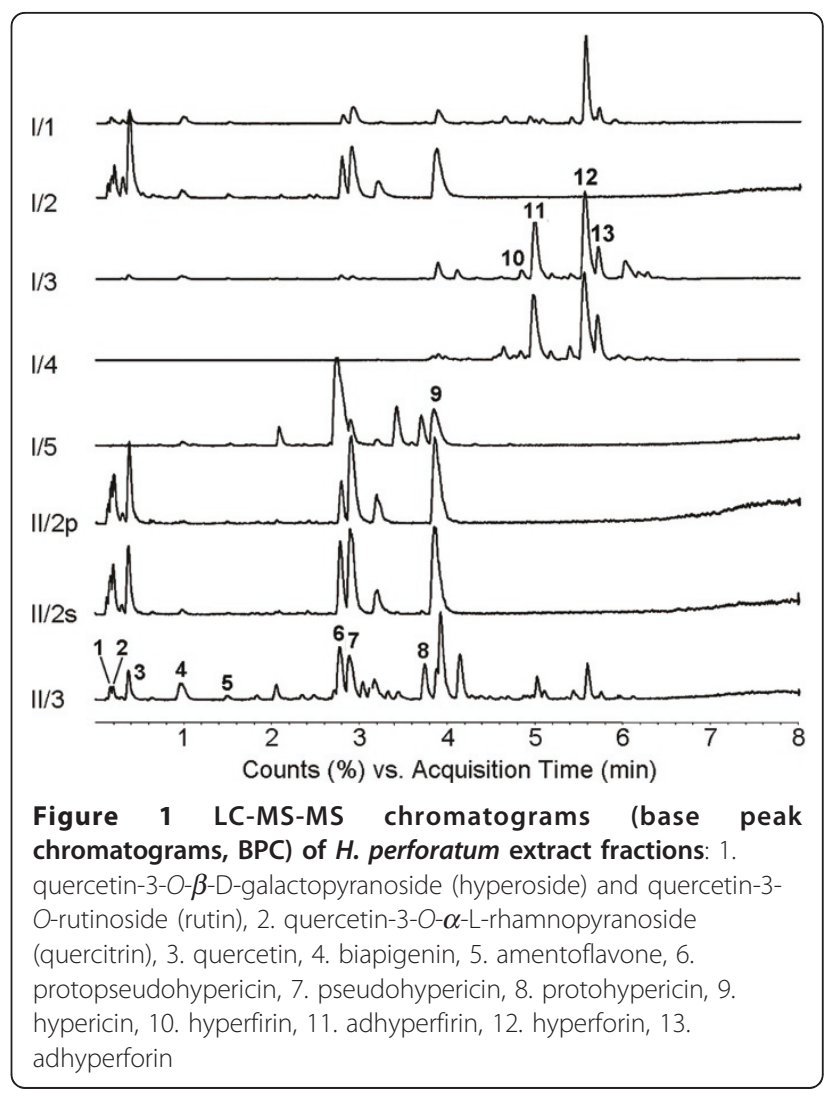

(Table 2). It is important to note that area percents, although useful for the comparison of the fractions' purity, neither directly correspond to the percentage composition in weight or amount, nor account for the total content of the compounds. Since there were no reference standards available, total peak areas (divided by the injected mass of extract) were used as a measure of the absolute content of the separate compound classes.

\section{Antioxidant activity}

All fractions were subjected to the antioxidant activity assays, with results given in Table 3 . Activities varied widely, from very high to moderate. All samples demonstrated ability to scavenge DPPH radicals, where most of them had $\mathrm{IC}_{50}$ value lower than the synthetic antioxidants BHT and BHA, except the fraction I/4. Superoxide anion scavenging was also observed, with $\mathrm{IC}_{50}$ values in range $1.86-32.4 \mu \mathrm{g} / \mathrm{mL}$ (the most active being $\mathrm{I} / 2$ and the least active $\mathrm{I} / 5$ ). Under the same experimental conditions, synthetic antioxidants BHT and BHA did not reach $50 \%$ inhibition due to their low solubility in the reaction medium (water). Inhibition of lipid peroxidation by extract fractions was also demonstrated, with $\mathrm{IC}_{50}$ higher (i.e. lower activity) than the synthetic antioxidants. Most of the fractions have shown scavenger activity in neutralizing NO radical. For fractions $\mathrm{I} / 2$ and 
Table 2 Relative abundances ${ }^{\mathrm{a}}$ of compound classes in analyzed fractions

\begin{tabular}{|c|c|c|c|c|c|c|c|}
\hline & \multicolumn{5}{|c|}{$\begin{array}{c}\text { Peaks } \\
0-2.5 \mathrm{~min}\end{array}$} & \multirow[t]{2}{*}{$\begin{array}{c}\text { Peaks } \\
\text { 2.5-4.7 } \text { min }^{\mathrm{c}}\end{array}$} & \multirow[t]{2}{*}{$\begin{array}{c}\text { Peaks } \\
\text { 4.7-6.5 min }\end{array}$} \\
\hline & Total $^{\mathrm{b}}$ & Phenolic acids & Flavonoid glycosides & Flavonoid aglycones & Biflavonoids & & \\
\hline $\mid-1$ & 16.2 & 0.430 & 2.72 & 4.41 & 6.14 & 31.8 & 68.7 \\
\hline $1-2$ & 37.2 & 1.30 & 6.49 & 20.3 & 3.02 & 49.1 & nd \\
\hline $1-3$ & 29.9 & $\mathrm{nd}^{\mathrm{e}}$ & 0.414 & 8.49 & 12.7 & 94.1 & 610 \\
\hline $\mid-4$ & 0.584 & nd & nd & nd & nd & 137 & 968 \\
\hline $1-5$ & 420 & nd & nd & 11.7 & 263 & 1000 & nd \\
\hline$\|-2 p$ & 17.9 & 0.866 & 5.77 & 7.67 & 0.496 & 36.9 & nd \\
\hline$\|-2 s$ & 17.3 & 0.743 & 5.83 & 6.70 & 1.40 & 41.5 & nd \\
\hline$\|-3$ & 31.4 & 0.257 & 4.50 & 7.75 & 14.2 & 119 & 28.4 \\
\hline
\end{tabular}

${ }^{a}$ given as peak areas divided by injected mass, normalized to 1000 for convenience

b including phenolic acids, flavonoids, biflavonoids and unidentified peaks

c mostly naphthodianthrones

d phloroglucinols

e not detected

$\mathrm{I} / 4$ it was not possible to determine $\mathrm{IC}_{50}$ values because neutralization of $50 \%$ NO radicals was not accomplished even with the highest concentrations of $22 \mu \mathrm{g} / \mathrm{mL}$ and $120 \mu \mathrm{g} / \mathrm{mL}$, respectively. Under the experimental conditions, synthetic antioxidants BHT and BHA did not reach $50 \%$ inhibition due to their low solubility in water.

\section{Discussion}

It has already been demonstrated $[10,17,19]$ that Hypericum perforatum extracts contain several classes of plant phenolics with a documented biological activity, including antidepressant phloroglucinols (hyperforin and its derivative adhyperforin), antiviral, antibacterial and photosensitizing naphthodianthrones (hypericin and pseudohypericin, as well as their precursors - protohypericin and protopseudohypericin), antioxidant flavonoids (mostly quercetin and kaempferol glycosides and aglycones, as well as biflavonoids), and phenolic acids (mostly isomeric caffeoylquinic acids). For the purpose of pharmaceutical products production, the preparation of the enriched extracts may be of interest. In this paper, it has been demonstrated that it is possible to obtain extracts with high levels of phloroglucinols (I/4) and naphthodianthrones (I/5) by using a relatively simple procedure.

The antioxidant activity of $H$. perforatum extracts is well known $[4,20]$ and is to be expected due to a high content of phenolic compounds. Although several publications were focused on this topic $[21,22]$, there is still a lack of conclusive evidence to determine which phenolics class is the most responsible for the antioxidant activity of Hypericum plants. Since the procedure described in this paper enabled the isolation of extract fractions enriched in different groups of phenolics, it was possible to establish the correlation between the extract composition and activity, and to distinguish structural features most important for scavenging and antioxidant properties. In addition, synergistic action of certain compounds is also to be expected.

Table 3 Results of antioxidant activity assays

\begin{tabular}{|c|c|c|c|c|c|c|}
\hline \multirow[b]{2}{*}{ Fractions } & \multicolumn{2}{|c|}{ DPPH } & \multirow{2}{*}{$\begin{array}{c}\mathrm{SO} \\
I C_{50}[\mu \mathrm{g} / \mathrm{mL}]\end{array}$} & \multirow{2}{*}{$\begin{array}{c}\mathrm{LP} \\
I C_{50}[\mu \mathrm{g} / \mathrm{mL}]\end{array}$} & \multirow{2}{*}{$\begin{array}{c}\mathrm{NO} \\
I C_{50}[\mu \mathrm{g} / \mathrm{mL}]\end{array}$} & \multirow{2}{*}{$\begin{array}{c}\text { FRAP } \\
{[\mathrm{mg} \mathrm{Fe} / \mathrm{g}]^{\mathrm{b}}}\end{array}$} \\
\hline & $I C_{50}[\mu \mathrm{g} / \mathrm{mL}]$ & {$[\mu \mathrm{g} / \mathrm{umol}]^{\mathrm{a}}$} & & & & \\
\hline $\mathrm{l} / 1$ & 1.55 & 69.2 & 11.1 & 2.25 & 32.8 & 80.0 \\
\hline $1 / 2$ & 1.09 & 48.7 & 1.86 & 7.92 & $>>22$ & 104.0 \\
\hline $1 / 3$ & 6.66 & 297 & 11.7 & 17.8 & 53.7 & 10.0 \\
\hline $1 / 4$ & 32.8 & 1464 & 20.6 & 2.33 & $>120$ & 7.00 \\
\hline $\mid / 5$ & 11.0 & 491 & 32.4 & 8.09 & 53.7 & 12.0 \\
\hline$\| / 2 s$ & 0.520 & 23.2 & 7.10 & 7.00 & 83.9 & 25.0 \\
\hline$\| / 2 p$ & 1.16 & 51.8 & 5.70 & 12.2 & 30.2 & 44.0 \\
\hline $11 / 3$ & 2.61 & 117 & 8.80 & 8.31 & 6.11 & 17.0 \\
\hline $\mathrm{BHT}$ & 8.28 & 369 & $\mathrm{n} / \mathrm{a}^{\mathrm{c}}$ & 0.859 & $\mathrm{n} / \mathrm{a}$ & 25.3 \\
\hline $\mathrm{BHA}$ & 12.4 & 553 & $\mathrm{n} / \mathrm{a}$ & 0.138 & $\mathrm{n} / \mathrm{a}$ & $\mathrm{n} / \mathrm{a}$ \\
\hline
\end{tabular}

\footnotetext{
${ }^{a}$ mass of extract (in $\mu \mathrm{g}$ ) needed for $50 \%$ neutralization of $1 \mu \mathrm{mol}$ of DPPH
}

${ }^{b}$ mass of $\mathrm{Fe}^{3+}$ that can be reduced by $1 \mathrm{~g}$ of $\mathrm{H}$. perforatum fraction.

c $50 \%$ inhibition not reached 
Since it is now recognized that there is no single test to evaluate antioxidant activities of the compounds with wide spectra of structures, modes of action, and physical and chemical properties [23], several different assays were employed as a part of our investigation.

DPPH (2,2-diphenyl-1-picrylhydrazyl) is a stable radical and is often used in assessment of the antioxidant activity. The free radical DPPH possesses a characteristic absorption at $517 \mathrm{~nm}$ (purple in color), which decreases significantly when exposed to radical-scavengers (due to hydrogen atoms transfer from antioxidant to DPPH). A lower absorbance at $517 \mathrm{~nm}$ indicates a higher radicalscavenging activity of extract [24]. In this assay, the ability of the investigated $H$. perforatum fractions to act as donors of hydrogen atoms or electrons in transformation of DPPH radical into its reduced form DPPH-H was investigated.

The activity observed is in a very good correlation with the composition, where the most active fractions are those rich in flavonoid glycosides (followed by minute amounts of phenolic acids) and poor in biflavonoids (amentoflavone, biapigenin): I/2, II/2p and II/2s. As opposed to this, fractions with high biflavonoids level and low flavonoid glycosides content $(\mathrm{I} / 3, \mathrm{I} / 5)$ exhibited significantly lower activity (i.e. higher $\mathrm{IC}_{50}$ values), and fraction I-4, practically devoid of flavonoids and phenolic acids, was the least active. It is notable that only flavonoid glycosides (with quercetin-3-O-rutinoside, galactoside and rhamnoside as the most abundant) seem to contribute significantly to radical-scavenging activity (given as $1 / \mathrm{IC}_{50}$ ), with Pearson's correlation coefficient $r$ $=0.778$ (0.922 if the outlier - II/2p - is excluded $)$, together with phenolic acids $(r=0.769$, or 0.929 without II/2p). These observations are only partially in agreement with the previous results [21]. While it would be expected for flavonoid aglycones to be more active than their 3-O-glycosides [23], no correlation was found between aglycones content and antioxidant activity as determined by DPPH test, and the activity of fractions I/3 and I/5 (rich in flavonoid and biflavonoid aglycones, poor in glycosides and phenolic acids) is low.

At the same time, no correlation was found between activity and content of naphthodianthrones and phloroglucinols, which is in accordance with the results of Silva et al. [23]. The low activity of biflavonoids present in Hypericum perforatum extracts was already documented [22] and is to be expected. These compounds, being dimers of apigenin, possess only 4'-hydroxyl on B-ring, while it is known that for maximum activity a catechol structure (3',4'-dihydroxylated benzene moiety), as present in quercetin, is required [21-23,25].

FRAP test is based on nonspecific reduction of $\mathrm{Fe}^{3}$ ${ }^{+}$-TPTZ into the blue $\mathrm{Fe}^{2+}$-TPTZ by the extract, which leads to an increased absorbance at $593 \mathrm{~nm}$. Results are presented as $\mathrm{mg}$ of $\mathrm{Fe}^{2+} / 1 \mathrm{~g}$ of dried fraction and represent the mass of $\mathrm{Fe}^{3+}$ that can be reduced by $1 \mathrm{~g}$ of $H$. perforatum fraction. Several important facts should be kept in mind when interpreting results of the test. First, it measures ability of a sample to participate in one-electron redox reactions, meaning that antioxidants with different mode of action (e.g. carotenoids, behaving as radical scavengers) will not be ignored. Second, only water-soluble antioxidants will react since the assay is performed in aqueous solution. Third, the reduction capacity determined in the absence of biomolecules as substrates does not necessarily reflect the antioxidant activity i.e. protective capabilities. Still, it can be a relevant factor in detoxification of reactive species such as $\mathrm{HOCl}$ and $\mathrm{ONOO}^{-}[26]$.

FRAP test has shown that $H$. perforatum fractions have the significant reduction potential, with fractions I/ $1, \mathrm{I} / 2$ and II/2p being more active than the synthetic antioxidant BHT. Taking into account the results of HPLC analysis, reduction capacity may be attributed mostly to the content of phenolic acids and flavonoid glycosides (although Pearson's correlation coefficients are quite low, 0.768 and 0.584 , respectively). The contribution of phloroglucinols to the total reduction capacity is negligible - two fractions rich in these compounds, I/ 4 and $I / 3$, exhibited the lowest capacity of all the samples examined. Their inactivity can be explained by the lack of easily oxidizable functional groups (such as phenolic moiety). The low activity is also observed for naphthodianthrones and biflavonoids, fractions with especially high content of these compounds (I/3, II/3, I/ $4, \mathrm{I} / 5)$ having the lowest reduction capacity.

In superoxide anion test the ability of $H$. perforatum fractions to neutralize superoxide anion radical $\left(\mathrm{O}_{2}{ }^{-}\right)$ was evaluated. While superoxide is intentionally produced by some cells (phagocytes) in order to combat infection, its presence is usually deleterious to organism, leading (if not degraded by superoxide dismutase) to degenerative processes and death. In the employed assay, $\mathrm{O}_{2}{ }^{-}$anion was generated in situ, by electrontransfer from NADH to $\mathrm{O}_{2}$ present in solution (a process analogous to NADPH oxidase- and NADH dehydrogenase-catalyzed generation in vivo) [20].

All investigated extract fractions exhibited dosedependent $\mathrm{O}_{2}{ }^{-}$scavenging activity. The weakest activity was again observed in samples rich in biflavonoids, naphthodianthrones and phloroglucinols but with low levels of other phenolics - I/4 and I/5. A high degree of correlation was found between the activity (expressed as $\left.1 / \mathrm{IC}_{50}\right)$ and the content of phenolic acids $(r=0.857)$, flavonoid aglycones $(r=0.791$, without the outlier I/5: $r$ $=0.926$ ), and sum of phenolic acids, flavonoid aglycones and glycosides $(r=0.883$, or 0.921 without fraction $\mathrm{I} / 5)$. These observations are in agreement with the empirical 
rule that a catechol moiety (present both in quercetin and in caffeoylquinic acid) is required for a maximum radical-scavenging activity [21-23,25].

One of the main detrimental effects of reactive radical species (especially $\mathrm{OH}$.) is lipid peroxidation (LP) i.e. oxidative degradation of lipids, leading to biological membranes damage and, possibly, to cell death or formation of mutagenic/carcinogenic products. The best known LP product is malondialdehyde (MDA) and it has been used most widely as a biomarker in various studies associated with lipid peroxidation. Determination of MDA may be problematic because of its high reactivity and water solubility, and it is therefore necessary to generate stable derivatives. One of the most commonly used is thiobarbituric acid adduct, which can be determined using spectrophotometry. In our research, linseed oil was used as a substrate for LP due to its high content of polyunsaturated fatty acids. LP of polyunsaturated fatty acids was triggered by $\mathrm{Fe}^{2+}$ and ascorbate (which, through Fenton reaction, generate $\mathrm{OH}$. radicals) [27].

All samples were able to inhibit lipid peroxidation in a dose-dependent manner, although not as efficient as synthetic antioxidants BHT and BHA. It is notable that fraction $\mathrm{I} / 4$, by far the poorest in flavonoids and phenolic acids and one of the least active in DPPH, FRAP, superoxide scavenging and NO scavenging assays, exhibited very strong LP-inhibiting activity, matched only by I/1. On the other hand, fraction II/2p, among the most active in mentioned assays, was second-to-weakest in this assay. It was not possible to correlate the test results with chemical composition of fractions. These results are in disagreement with those of Silva et al. [21], which implied flavonoids as main anti-LP constituents of Hypericum perforatum extracts.

It should be noted that the effects of plant phenolics on $\mathrm{Fe}^{2+}$ /ascorbate-induced lipid peroxidation are complex. It is known that flavonoids, especially those with catechol-like substitution on B ring and either 4-oxo-3hydroxy or 4-oxo-5-xydroxy, are efficient in inhibiting LP, both through radical scavenging and through chelation of iron ions. On the other hand, in the presence of transition metal ions, flavonoids also exhibit pro-oxidant effect [27], continuously reducing these ions and thus enabling them to generate $\mathrm{OH}$. via Fenton reaction. Biflavonoids, while being very poor radical scavengers, are proven to inhibit LP with similar potency as flavonoid glycosides, probably through iron chelation [22], while naphthodianthrones and phloroglucinols are not active [21].

Finally, nitric oxide scavenging ability was also assayed for all fractions. While NO is normally produced in organism as a messenger and as a part of immune response, its reaction with $\mathrm{O}_{2}^{-}$produces highly reactive peroxynitrite that can damage various biomolecules. Thus, the ability of extracts to neutralize NO and $\mathrm{ONOO}^{-}$can have beneficial effects.

While all investigated extract fractions demonstrated dose-dependent scavenging of $\mathrm{NO}$, for most of them $\mathrm{IC}_{50}$ values were high (or, in the case of $\mathrm{I} / 2$ and $\mathrm{I} / 4$, $50 \%$ neutralization was not accomplished within the examined concentration range). As with lipid peroxidation assay, it was not possible to attribute the NO scavenging activity to any particular class of phenolics.

\section{Conclusion}

In conclusion, by using fractionation combined with assays and LC-MS analysis, it was possible to identify compound classes responsible for $H$. perforatum antioxidant activity. It was demonstrated that it can mostly be attributed to flavonoid glycosides and phenolic acids (chlorogenic acid), while phloroglucinols (lacking polyphenolic structure), biflavonoids (lacking catechol moiety) and naphthodianthrones showed no significant activity.

\section{Experimental \\ Chemicals}

Following reagents were purchased from Sigma-Aldrich Chem, Steinheim, Germany: BHT, BHA, NADH, phenazine methosulfate (PMS). Sulfanilamide, 2,2-diphenyl-1picrylhydrazyl (DPPH), and 2-thiobarbituric acid (TBA) were obtained from Fluka Chemie GmbH (Buchs, Switzerland). Trichloroacetic acid was purchased from LachNer (Neratovice, Czech Republic), nitroblue tetrazolium (NBT) from Alfa Aesar (Karlsruhe, Germany), and sodium nitroprusside (SNP) from Reanal (Budapest, Hungary). $N$-(1-naphthyl) ethylenediamine dihydrochloride (NEDA), ascorbic acid, 2,4,6-tripyridil-s-triazine (TPTZ), and formic acid was acquired from Merck, Darmstadt, Germany. Tween-80 and gradient-grade acetonitrile were obtained from J. T. Baker (Deventer, Netherlands). Linseed oil is prepared by solvent extraction of linseed, and fatty acids composition is determined by GC-MS to be: linolenic $69.7 \%$, linoleic $13.5 \%$, stearic $9.4 \%$ and palmitic $7.4 \%$. FRAP reagent was made by mixing $50 \mathrm{ml}$ of acetate buffer $(0.3 \mathrm{~mol} / \mathrm{L}, \mathrm{pH}=3.6)$, $5 \mathrm{~mL}$ of $\mathrm{FeCl}_{3} \cdot 6 \mathrm{H}_{2} \mathrm{O}(20 \mathrm{mmol} / \mathrm{L})$ and $5 \mathrm{~mL}$ of TPTZ solution $(10 \mathrm{~mol} / \mathrm{L}$ in $40 \mathrm{mmol} / \mathrm{L} \mathrm{HCl})$. TBA reagent was prepared by dissolving $3 \mathrm{~g}$ of TBA, $120 \mathrm{~g}$ of trichloroacetic acid and $10.4 \mathrm{~mL}$ of perchloric acid in water and filling up to $1 \mathrm{~L}$. All chemicals were reagent grade, unless otherwise stated.

\section{Plant material}

Hypericum perforatum L. (Clusiaceae) samples were collected mid July 2007 from the mountain Čemernik, south of Serbia, at blooming stage. The voucher 
specimen (No. 11340) is deposited at the Herbarium of the Institute of Botany and Botanical Garden "Jevremovac", Faculty of Biology, University of Belgrade (BEOU). Top flowering parts were used for extract preparation (80\% flowers and $20 \%$ rest of the plant). Plant material was dried and powdered, and dried again at $105^{\circ} \mathrm{C}$ by steam heating.

\section{Extraction}

The extraction and fractionation were performed using third party-developed procedure (patent pending). Dried and powdered plant material was depigmentated using chloroform extraction for $96 \mathrm{~h}$ and, after vacuum-drying, extracted for $72 \mathrm{~h}$ with eight-fold amount of methanol. Both extractions were carried out at temperatures near the boiling points of the respective solvents. The process yielded $6-7 \%$ of raw extract on dry weight basis. After removal of carotenoids by petroleum ether extraction and glycosides by ethyl acetate/water partitioning, the residue was fractionated using Sephadex LH-20 column to give five fractions (based on colour and UV/VIS absorption spectra), labelled I/1 to I/5 for the first batch of plant material, and II/ 1 to II/5 for the second batch (of which, only sub-fractions II/2s and II/2p, and fraction II/3 were analyzed).

For the purpose of LC analysis and antioxidant assays, all fractions were dissolved in methanol (except fraction $\mathrm{I} / 4$, for which iso-propanol was used, due to low solubility in methanol).

\section{LC-MS analysis}

Chemical composition of fractions was determined by rapid resolution liquid chromatography with mass selective detection, using Agilent Technologies 1200 Series liquid chromatograph coupled with Agilent Technologies 6410B Series triple-quad (QQQ) mass spectrometer. Components were separated using reversed-phase Zorbax SB-C18 $30 \mathrm{~mm} \times 2.1 \mathrm{~mm} \times 3.5 \mu \mathrm{m}$ column (Agilent Technologies), held at $50^{\circ} \mathrm{C}$. The mobile phase was delivered in gradient mode (0 min 25\% B, 6 min 100\% B, 8 min $100 \%$ B, solvent $\mathrm{A}$ being $0.1 \%$ aqueous formic acid with $10 \mathrm{mmol} / \mathrm{L} \mathrm{CH}_{3} \mathrm{COONH}_{4}$, and solvent $\mathrm{B}$ being acetonitrile), with flow rate of $1 \mathrm{~mL} / \mathrm{min}$. Injection volume was $1 \mu \mathrm{L}$, except for sample I/5, for which 0.1 $\mu \mathrm{L}$ was used to improve peak shape. Eluted components were ionized by electrospray ion source (ESI), using $\mathrm{N}_{2}$ for nebulization (pressure of $35 \mathrm{psi}$ ) and drying (flow of $9 \mathrm{~L} / \mathrm{min}$, temperature of $350^{\circ} \mathrm{C}$ ). Capillary voltage was $4000 \mathrm{~V}$ and fragmentor voltage $80 \mathrm{~V}$. To increase the sensitivity, lower the noise, and simplify the spectra, negative ionization was used. Generated $[\mathrm{M}-\mathrm{H}]^{-}$ions were analyzed using MS2Scan mode, in $\mathrm{m} / \mathrm{z}$ range 200$800 \mathrm{Da}$.

\section{DPPH assay}

Selected concentrations (ranging 0.001-12 mg/mL) of $H$. perforatum fractions $(10 \mu \mathrm{L})$ were mixed with $190 \mu \mathrm{L}$ of methanol and $100 \mu \mathrm{L}$ of methanolic solution containing DPPH radicals $(67.2 \mu \mathrm{mol} / \mathrm{L})$. Absorption at $515 \mathrm{~nm}$ was measured by the microplate reader (Multiskan Spectrum, Thermo Corporation) after $60 \mathrm{~min}$ of incubation at room temperature. The radical-scavenging capacity $(R S C)$ was calculated using the equation:

$$
R S C=100-\frac{\left(A_{\text {average }}-A_{\text {corr }}\right)}{A_{\text {control }}} \cdot 100
$$

where $A_{\text {average }}$ is absorbance of probe, at a given sample concentration level (average of three probes); $A_{\text {corr }}$ is the correction or the absorbance of the extract alone (without reagents), and $A_{\text {control }}$ is the absorbance of the reagent (DPPH radical) without the extract. The extract concentration in reaction mixture, resulting in $50 \%$ inhibition of DPPH radicals $\left(\mathrm{IC}_{50}\right)$, was calculated from the $R S C$ vs. extract concentration curve.

\section{FRAP (ferric reducing ability of plasma)}

Selected concentrations (ranging 0.001-12 mg/mL) of $H$. perforatum fractions $(10 \mu \mathrm{L})$ were treated with $300 \mu \mathrm{L}$ of FRAP reagent in triplicate. Correction (absorbance of the untreated extract) and control (absorbance of the FRAP reagent) were also measured. After $6 \mathrm{~min}$ of incubation, the absorbance was measured at $593 \mathrm{~nm}$ by the microplate reader (Multiskan Spectrum, Thermo Corporation). The calibration curve was constructed using the aqueous solution of $\mathrm{Fe}^{2+}$ in series of concentration ranging $50-1000 \mu \mathrm{mol} / \mathrm{L}$. The results were given as $\mathrm{mg}$ of $\mathrm{Fe}^{2+}$ per gram of dry extract.

\section{Superoxide anion test}

Selected concentrations (ranging 0.001-12 mg/mL) of $H$. perforatum fractions $(10 \mu \mathrm{L})$ were mixed with $40 \mu \mathrm{L}$ of $144 \mu \mathrm{mol} / \mathrm{L}$ NBT, $20 \mu \mathrm{L}$ of $677 \mu \mathrm{mol} / \mathrm{L} \mathrm{NADH}, 20 \mu \mathrm{L}$ of $60 \mu \mathrm{mol} / \mathrm{L}$ PMS and $220 \mu \mathrm{L}$ of $\mathrm{pH} 8.3$ buffer in triplicate. Correction (absorbance of untreated extract) and control (absorbance of reagents without extract) were also measured. After $5 \mathrm{~min}$ of incubation absorbance was measured at $560 \mathrm{~nm}$ by the microplate reader (Multiskan Spectrum, Thermo Corporation). The values of $R S C$ and $\mathrm{IC}_{50}$ were calculated in the same manner as in DPPH test.

\section{Inhibition of NO radical}

Selected concentrations (ranging 0.001-12 mg/mL) of $H$. perforatum fractions $(10 \mu \mathrm{L})$ were mixed with $75 \mu \mathrm{L}$ of SNP and $75 \mu \mathrm{L}$ of phosphate buffer $(0.067 \mathrm{~mol} / \mathrm{L}, \mathrm{pH}=$ 7.4) in triplicate. Correction (absorbance of untreated extract) and control (absorbance of reagents without 
extract) were also measured. Samples were incubated under a fluorescent lamp for $1 \mathrm{~h}$. After the incubation, in each well $150 \mu \mathrm{L}$ of Griess reagent was added and the absorbance was measured at $546 \mathrm{~nm}$ by microplate reader (Multiskan Spectrum, Thermo Corporation). The values of $R S C$ and $\mathrm{IC}_{50}$ were calculated in the same manner as in DPPH test.

\section{Lipid peroxidation}

As a substrate for lipid peroxidation, linseed oil (prepared in-laboratory by hexane extraction of linseed) was emulsified in phosphate buffer $(0.035 \% \mathrm{v} / \mathrm{v}$ solution) with addition of Tween- $80(0.25 \% \mathrm{v} / \mathrm{v}$ solution $)$ as an emulsifier. Selected concentrations (ranging $0.0001-1.2 \mathrm{mg} / \mathrm{mL})$ of $H$. perforatum fractions $(10 \mu \mathrm{L})$, each in triplicate, were mixed with $20 \mu \mathrm{L}$ of 1.875 $\mathrm{mmol} / \mathrm{L} \mathrm{FeSO}_{4}$ and $20 \mu \mathrm{L}$ of ascorbate $(15.4 \mu \mathrm{g} / \mathrm{mL})$. The control and corrections were also made as previously defined. All probes were incubated at $37^{\circ} \mathrm{C}$ for $1 \mathrm{~h}$, after which $200 \mu \mathrm{L}$ of $0.1 \mathrm{~mol} / \mathrm{L}$ EDTA was added to bind the $\mathrm{Fe}^{2+}$, thus stopping the reaction. TBA reagent $(2 \mathrm{~mL})$ was added, and the mixture was heated in boiling water bath for $15 \mathrm{~min}$ to form a stable, colored derivative with MDA [28].

After that, probes were centrifuged for $15 \mathrm{~min}$ at 3700 $\mathrm{rpm}$ and the absorbance was measured at $532 \mathrm{~nm}$. The values of $R S C$ and $\mathrm{IC}_{50}$ were calculated in the same manner as in DPPH test.

\section{Abbreviations}

BHT: 3,5-di-tert-butyl-4-hydroxytoluene; BHA: 2-tert-butyl-4-hydroxyanisole; DPPH: 2,2-diphenyl-1-picrylhydrazyl; ESI: electrospray ionization; NBT: nitroblue tetrazolium; NEDA: N-(1-naphthyl) ethylenediamine dihydrochloride; PMS: phenazine methosulfate; SNP: sodium nitroprusside; TBA: 2thiobarbituric acid; TPTZ: 2,4,6-tripyridil-s-triazine.

\section{Acknowledgements}

This research is funded by Serbian Ministry of Science and Technological Development research grant Nr. 172058. and Provincial Secretariat for Sciences and Technological Development (grants 2011-2014).

\section{Author details}

${ }^{1}$ Department of Chemistry, Biochemistry and Environmental Protection, University of Novi Sad Faculty of Sciences, Trg Dositeja Obradovića 3, Novi Sad, Serbia. ${ }^{2}$ BIOSS, Bulevar Oslobođenja 401i, Belgrade, Serbia.

\section{Authors' contributions}

SSP performed sample preparations and fractionation of plant material. DZO developed LC/MS method, performed qualitative phenolics analysis, and contributed to the results interpretation. MMF and EĐJ performed antioxidant assays. NMMD coordinated the study, participated in its design and contributed to drafting manuscript. All authors have read and approved the final manuscript.

\section{Competing interests}

The authors declare that they have no competing interests.

Received: 13 April 2011 Accepted: 25 June 2011

Published: 25 June 2011

\section{References}

1. Luo L, Sun Q, Mao YY, Lu YH, Tan RX: Inhibitory effects of flavonoids from Hypericum perforatum on nitric oxide synthase. J Ethnopharmacol 2004, 93:221-225.

2. Jakovljević $V$, Popović M, Mimica-Dukić N, Sabo A, Gvozdenović Lj: Pharmacodynamic study of Hypericum perforatum L. Phytomedicine 2000, 7:449-453.

3. Popović M, Jakovljević V, Mimica-Dukić N, Kaurinović B, Cebović T: Effects of different extracts of Hypericum perforatum $\mathrm{L}$. on the $\mathrm{CCl}_{4}$-induced hepatotoxicity in rats. Oxid Commun 2002, 25:273-278.

4. Radulović N, Stankov-Jovanović V, Stojanović G, Šmelcerović A, Spiteller M, Asakawa Y: Screening of in vitro antimicrobial and antioxidant activity of nine Hypericum species from the Balkans. Food Chem 2007, 10:315-21.

5. Spiteller M, Ozen T, Šmelcerović A, Zuehlke S, Mimica-Dukić N: Phenolic constituents and the in vitro antioxidant activity of the flowers of Hypericum venustum. Fitoterapia 2008, 79:191-193.

6. Breyera A, Elstnerb M, Gillessenc T, Weiserd D, Elstner E: Glutamate-induced cell death in neuronal HT22 cells is attenuated by extracts from St. John's wort (Hypericum perforatum L.). Phytomedicine 2007, 14:250-255.

7. Meruelo D, Lavie G, Lavie D: Therapeutic agents with dramatic antiretroviral activity and little toxicity at effective doses: aromatic polycyclic diones hypericin and pseudohypericin. PNAS 1988, 85:5230-5234.

8. Gioti E, Fiamegos Y, Skalkos D, Stalikas C: Improved method for the in vitro assessment of antioxidant activity of plant extracts by headspace solid-phase microextraction and gas chromatography-electron capture detection. J Chromatogr A 2007, 1152:150-155.

9. Ivetić V, Popović M, Mimica-Dukić N, Barak O, Pilija V: St John's wort (Hypericum perforatum L.) and kindling epilepsy in rabbit. Phytomedicine 2002, 9:496-499.

10. Brolis M, Gabetta B, Fuzzati N, Pace R, Panzeri F, Peterlongo F: Identification by high-performance liquid chromatography-diode array detection-mass spectrometry and quantification by high-performance liquid chromatography-UV absorbance detection of active constituents of Hypericum perforatum. J Chromatogr A 1998, 825:9-16.

11. Mauri P, Pietta P: High performance liquid chromatography/electrospray mass spectrometry of Hypericum perforatum extracts. Rapid Commun Mass Sp 2000, 14:95-99.

12. Piperopoulos G, Lotz R, Wixforth A, Schmierer Th, Zeller KP: Determination of naphthodianthrones in plant extracts from Hypericum perforatum $\mathrm{L}$. by liquid chromatography-electrospray mass spectrometry. J Chromatogr B 1997, 695:309-316.

13. Poutaraud A, Lobstein A, Girardin P, Weniger B: Improved Procedure for the Quality Control of Hypericum perforatum L. Phytochem Anal 2001, 12:355-362.

14. Ganzera M, Zhao J, Khan IA: Hypericum perforatum - chemical profiling and quantitative results of St. John's wort products by an improved high-performance liquid chromatography method. J Pharm Sci-US 2002, 91:623-630.

15. Tolonen A, Hohtola A, Jalonen J: Fast High-performance Liquid Chromatographic Analysis of Naphthodianthrones and Phloroglucinols from Hypericum perforatum extracts. Phytochem Anal 2003, 14:306-309.

16. Liu F, Pan Ch, Drumm P, Ang CYW: Liquid chromatography-mass spectrometry studies of St. John's wort methanol extraction: active constituents and their transformation. J Pharmaceut Biomed 2005, 37:303-315.

17. Tatsis EC, Boeren S, Exarchou V, Vervoort ANTJ, Gerothanassis IP: Identification of the major constituents of Hypericum perforatum by LC/ SPE/NMR and/or LC/MS. Phytochemistry 2007, 68:383-393.

18. Bonkanka CX, Smelcerovic A, Zuehlke S, Rabana RM, Spiteller M, SánchezMateo CC: LC-MS analysis and anti-oedematogenic activity of Hypericum grandifolium Choisy (Hypericaceae). Planta Med 2008, 74:719-725.

19. Huck CW, Abel G, Popp M, Bonn GK: Comparative analysis of naphthodianthrone and phloroglucine derivatives in St. John's Wort extracts by near infrared spectroscopy, high-performance liquid chromatography and capillary electrophoresis. Anal Chim Acta 2006, 580:223-230

20. Sagratini G, Ricciutelli M, Vittori S, Öztürk N, Öztürk Y, Maggi F: Phytochemical and antioxidant analysis of eight Hypericum taxa from Central Italy. Fitoterapia 2008, 79:210-213. 
21. Silva BA, Ferreres F, Malva JO, Dias ACP: Phytochemical and antioxidant characterization of Hypericum perforatum alcoholic extracts. Food Chem 2005, 90:157-167.

22. Silva BA, Malva JO, Dias ACP: St. John's Wort (Hypericum perforatum) extracts and isolated phenolic compounds are effective antioxidants in several in vitro models of oxidative stress. Food Chem 2008, 110:611-619

23. Apak R, Güçlü K, Demirata B, Özyürek M, Çelik SE, Bektaşoğlu B, Berker KI, Özyurt D: Comparative Evaluation of Various Total Antioxidant Capacity Assays Applied to Phenolic Compounds with the CUPRAC Assay. Molecules 2007, 12:1496-1547.

24. Sánchez-Moreno C: Methods used to evaluate the free radical scavenging activity in foods and biological systems. Food Sci Technol Int 2002, 8:121-137.

25. Rice-Evans CA, Miller NJ, Paganga G: Structure-antioxidant activity relationships of flavonoids and phenolic acids. Free Radic Biol Med 1996, 20:933-956.

26. Macdonald-Wicks LK, Wood LG, Garg ML: Methodology for the determination of biological antioxidant capacity in vitro: a review. J Sci Food Agric 2006, 86:2046-2056.

27. Laguerre M, Lecomte J, Villeneuve P: Evaluation of the ability of antioxidants to counteract lipid oxidation: Existing methods, new trends and challenges. Prog Lipid Res 2007, 46:244-282.

28. Božin B, Mimica-Dukić N, Samojlik I, Anačkov G, Igić R: Phenolics as antioxidants in garlic (Allium sativum L., Alliaceae). Food Chem 2008, 111:925-929.

doi:10.1186/1752-153X-5-34

Cite this article as: Orčić et al: Antioxidant activity relationship of phenolic compounds in Hypericum perforatum L.. Chemistry Central Journal 2011 5:34.

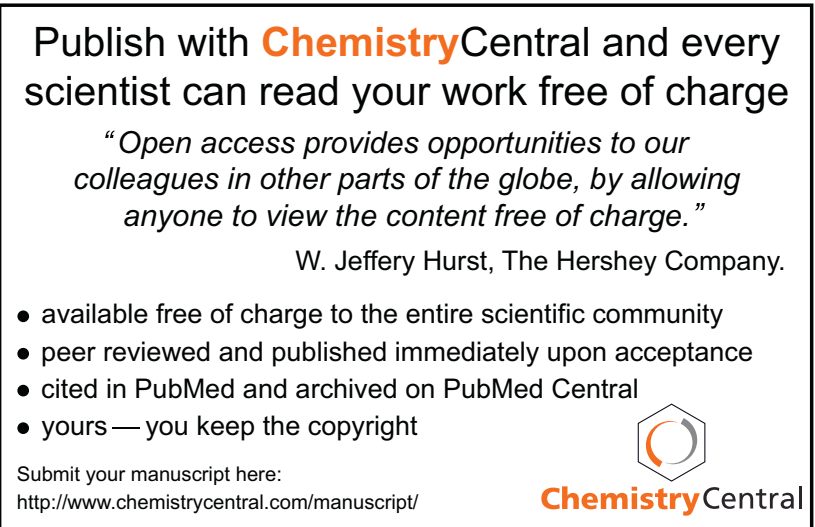

